\title{
Notions d'esthétique dans les traités arabes sur la musique
}

Amnon Shiloah

\section{OpenEdition}

\section{Journals}

Édition électronique

URL : http://journals.openedition.org/ethnomusicologie/1335

ISSN : 2235-7688

\section{Éditeur}

ADEM - Ateliers d'ethnomusicologie

Édition imprimée

Date de publication : 31 décembre 1994

Pagination : $51-58$

ISBN : 2-8257-0503-9

ISSN : 1662-372X

\section{Référence électronique}

Amnon Shiloah, « Notions d'esthétique dans les traités arabes sur la musique », Cahiers

d'ethnomusicologie [En ligne], 7| 1994, mis en ligne le 03 janvier 2012, consulté le 19 avril 2019. URL: http://journals.openedition.org/ethnomusicologie/1335

Ce document a été généré automatiquement le 19 avril 2019

Tous droits réservés 


\title{
Notions d'esthétique dans les traités arabes sur la musique
}

\author{
Amnon Shiloah
}

1 Les jugements sur la musique que nous relevons dans les anciens écrits arabes nous confrontent d'emblée à une image fort complexe où s'entremêlent des approches diverses. Nous y trouvons bien entendu maintes considérations mettant en relief l'extraordinaire pouvoir éthique de la musique, aussi bien que ses valeurs en tant qu'art anagogique, c'est-à-dire sa capacité à diriger l'esprit vers le monde supérieur et le symbolisme universel. Nous pouvons y rencontrer également, avec non moins de fréquence, une approche qui admet l'amour de l'art musical en soi, à travers sa palette de combinaisons sonores et le plaisir qu'elle suscite. C'est sur cette sorte d'expérience esthétique que nous focaliserons ici notre attention, sans toutefois minimiser l'importance de l'approche éthique.

2 Disons-le tout de suite, il n'est pas dans notre intention de nous référer à une quelconque théorie esthétique, mais plutôt de donner la parole aux représentants de la culture examinée, étant convaincu que les idées glanées dans la littérature reflètent des valeurs, des normes, des modes de pensée et des formes d'expression qui conditionnent l'approche du style musical du groupe envisagé.

3 Les témoignages sur lesquels reposent nos observations appartiennent à cette catégorie qu'Alan Merriam qualifie de verbal behaviour qui, selon lui, met en valeur des concepts. Merriam ajoute: «But in this case applied specifically to what people say about music structures and the criteria which surround it » (1964: 114). Ceci, ainsi que les critères de jugement concernant l'interprétation musicale feront l'objet essentiel de cet exposé. Dans le cadre de ces propos préliminaires, il convient de s'attarder brièvement sur le constat suivant, établi par l'éminent spécialiste de la musique arabe, H. G. Farmer, dans l'aperçu général « The Music of Islam »: " Aesthetics, the science of the beautiful, did not exist in islamic conceptions. The chief reason for this was that the Orient [...] put great trust in the doctrine of the ta'thir (influence) in music, a dogme called by the Greeks the ethos " (Farmer 1957: 433). Je dois avouer que, déjà à la première lecture, cette façon de voir m'a intrigué, sans néanmoins diminuer mon admiration pour ce savant pionnier. Mon 
étonnement était d'autant plus grand que ce même spécialiste m'avait recommander d'étudier l'ouvrage manuscrit d'Al-Ḥasan Al Kātib (1972) qui, selon lui, constituait une contribution considérable à la musicologie arabe. Or, cet ouvrage, symboliquement intitulé La perfection des connaissances musicales, recherche, suivant les buts assignés par son auteur, l'idéal de perfection de l'art musical, en faisant appel à des témoignages de ses contemporains et de ses prédécesseurs. Dans cet ouvrage, l'auteur nous propose maintes observations et considérations que nous pouvons qualifier d'expériences esthétiques.

Cette méthode empirique et parfois même anecdotique émane du fait qu'elle est liée à la pratique et prend sa forme essentielle dans les réactions instantanées. A cela, il faut ajouter le facteur important de la transmission orale, avec toutes les limites qu'elle impose à l'auditeur comme au critique. La nature du jugement a donc été dictée par ce phénomène, comme pour la poésie arabe dont la survie était assurée dans une large mesure par le truchement de sa récitation en public; le parallélisme entre ces deux arts va encore plus loin, comme nous le verrons par la suite. Il est dû non seulement à la prédominance de la musique vocale qui détermine l'union intime entre poésie et mélodie, mais aussi à la parenté entre théorie littéraire et esthétique musicale, qui voient le jour à la même époque - fin du IX ${ }^{\mathrm{e}}$ siècle et surtout $\mathrm{X}^{\mathrm{e}}$ siècle. Est-ce une pure coïncidence, ou bien s'agit-il d'un rapport étroit, voire même essentiel, qui les relie sur le plan conceptuel? C'est bien la seconde éventualité qui semble s'imposer.

\section{Théorie littéraire et esthétique musicale}

Dans un ouvrage publié en 1982, intitulé Beyond the Line («Au-delà de la ligne», qui désigne ici le vers en poésie), l'orientaliste hollandais van Gelder présente une analyse de critiques littéraires arabes classiques mettant l'accent sur le problème de la cohérence et de l'unité dans cette poésie (1982). Le titre de l'ouvrage s'inspire du fait qu'en règle générale c'est bien la ligne isolée qui constitue le centre d'intérêt des critiques, se reflétant dans les théories littéraires écrites qui commencent à paraitre dès la deuxième moitié du IXe siècle. Ces théories continuent à faire valoir les artifices rhétoriques, le badī c, concept traduisant des idées et expressions inédites, insolites extraordinaires, ainsi que des aspects relatifs à la grammaire, à la lexicographie. Ce sont là des critères de jugement essentiels. Autour de cette molécularité caractérisant la conception d'une oeuvre poétique et son jugement, van Gelder examine la question de cohérence et d'unité telle qu'elle s'exprime dans les sources arabes.

6 L'unité et la cohérence, dans un poème polythématique, sont en partie obtenues par l'enchaînement des thèmes qui doivent être assurés par des transitions élégantes et soignées. Cependant, signale van Gelder, qui adopte une solution intermédiaire, les relations entre les parties composant un poème, aussi bien que la cohérence et la totalité de l'oeuvre, sont généralement moins importantes pour le poète que le souci de divertir, ou de frapper l'imagination du lecteur ou de l'auditeur. A cet effet, il faut aussi tenir compte du fait que, dans toutes les formes de la poésie, réciter et écouter étaient bien plus importants qu'écrire ou lire. D'où la difficulté de s'en faire une idée complète à l'audition. Le plaisir que l'on peut tirer provient souvent de l'effet frappant produit par un fragment ou un détail insolite, par l'élégance et par la belle construction d'une phrase, par la formulation inédite d'une pensée. La combinaison des thèmes et motifs dans un poème, leur compatibilité et leur interaction ne préoccupent pas les critiques aussi longtemps que des contradictions choquantes ou des dissonances déplaisantes ne 
viennent porter atteinte à l'ensemble, ressenti de façon sous-jacente. En effet, comme le remarque van Gelder: "Une séquence particulière d'associations peut paraitre évidente et par conséquent cohérente à une personne mais pas à une autre, à une communauté littéraire, mais pas à une autre ».

7 En conclusion, van Gelder dit qu'étant donné que le sens du mot " œuvre » ou " poème " est en quelque sorte fluide dans la littérature arabe, nous devons prendre en considération le but que se propose le poète, les méthodes, le goût et les critères employés par les critiques en jugeant la poésie classique. "Etudier ces théories ne constitue pas une activité complémentaire pour l'étudiant, mais l'étude de la poésie ellemême » (1982: 208).

Ce détour par les chemins de la poésie arabe classique n'est aucunement fortuit. Car, quand il s'agit de l'alliance entre poésie et mélodie, nous retrouvons dans l'esthétique musicale, non seulement des règles et conceptions analogues, mais aussi des similarités de points de vue au sujet de l'« œuvre » musicale et de son interprétation. Bien sûr, tout en puisant dans les mêmes sources conceptuelles, l'esthétique musicale a développé des normes et critères de jugement qui lui sont propres, surtout dans le cadre de l'interprétation musicale en public. Dans ce cas précis, l'oralité joue un rôle encore plus évident que dans la lecture publique de la poésie.

\section{Critères concernant la composition musicale}

En discutant des critères de la composition musicale ou plutôt du chant, les théoriciens et les critiques font toujours état de deux constituants: le poème et la mélodie dans leur parfaite association. Tout en admettant l'éventualité d'une espèce de mélodie indépendante et sans paroles, dont l'effet se résume à flatter le sens auditif, les théoriciens sont unanimes à penser que la perfection n'est atteinte que lorsque la mélodie est associée à un texte poétique. Dans ce cas, la mélodie puise, d'une part, son sens dans les paroles auxquelles elle est associée, et, d'autre part, elle prolonge leur sens, les renforce et augmente la beauté du vers. Même dans cette association, il s'agit toujours de vers isolés sur lesquels sont calquées la structure et la modalité de la mélodie. Constituant ainsi une entité inséparable, les critères d'analyse visent à la fois la versification, la prosodie et l'éloquence, ainsi que les aspects purement mélodiques.

Dans un passage concernant la composition musicale, Al-Kātib écrit: «Il est préférable dans la répartition d'une mélodie que la phrase mélodique du second vers soit pareille dans toutes ses modalités à celle du premier, sauf si elle est une réplique à l'octave. » (1972: 111). La réplique à l'octave est en effet un artifice esthétique fort apprécié dans l'interprétation musicale. Al-Kātib ajoute, dans le cadre de sa définition, que les coupures de phrases mélodiques devraient coïncider avec celles des pieds métriques et des hémistiches; que les vocalises devraient être placées sur les coupures et de préférence en fin d'hémistiche ou de vers. A propos des vocalises et des ornements, Al-Kātib mentionne les consonnes sur lesquelles il conviendrait de placer de préférence vocalises et sons prolongés. 
Tuğra du Sultan Soliman le Magnifique. Turquie, période ottomane, vers 1555-1560.

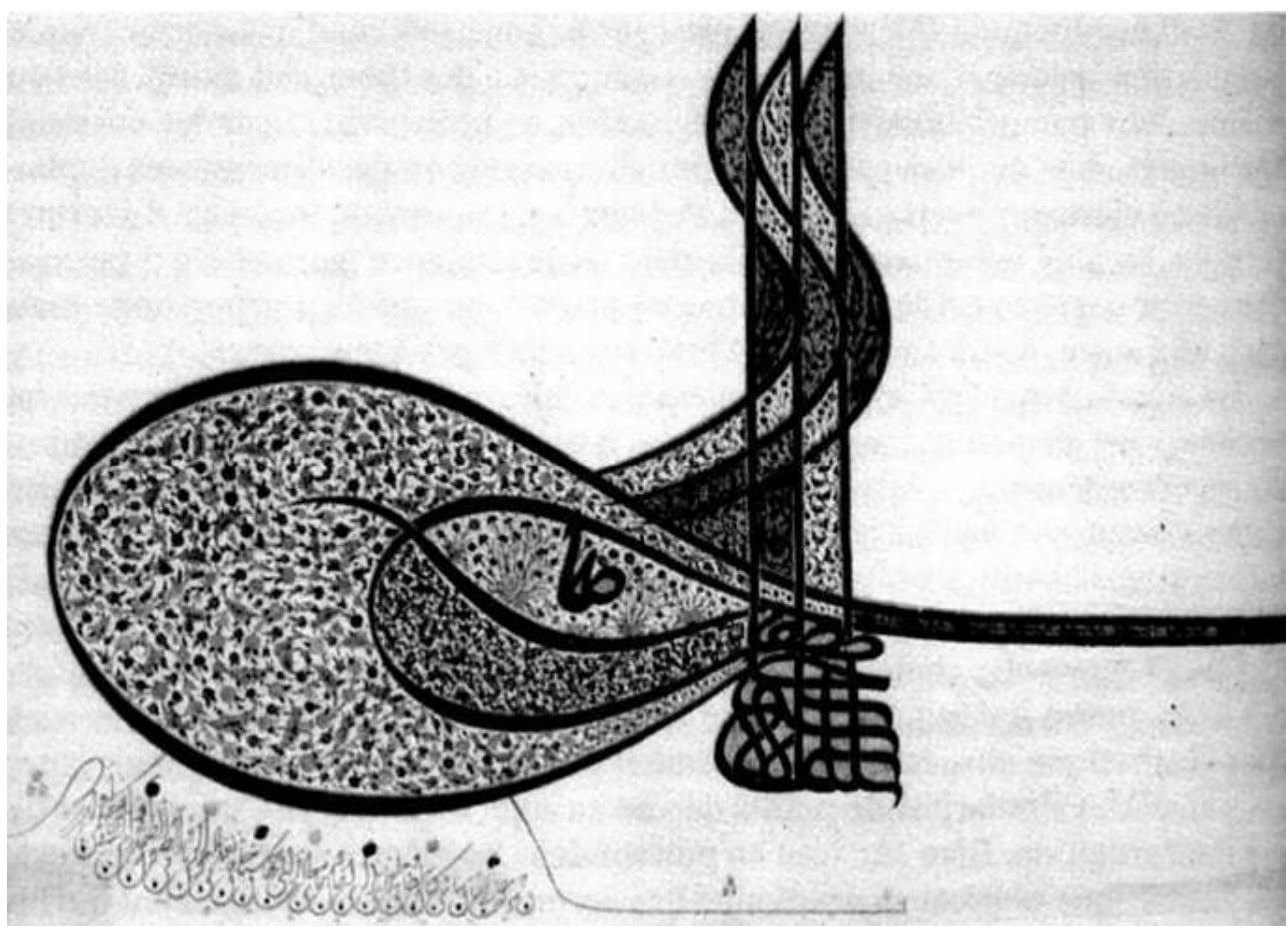

Calligraphie à l'encre, colorée et dorée.

Coll. Metropolitan Museum of Arts, New York.

11 Parmi les menus détails qui caractérisent les composants mélodiques, il y a ce qu'AlFārābì qualifie de modalités de notes, qui donnent à la mélodie élégance, beauté et toute une gamme d'expressions (d'Erlanger 1935, vol. II: 89). Dans le même ordre d'idées, AlKātib nous fournit des renseignements précieux sur ce qu'il appelle « choses qui rendent les mélodies plus agréables à l'oreille», par exemple "que les notes de longue durée soient chevrotées et tremblées; que les notes ornées soient douces; que certaines notes soient serrées entre les lèvres et que d'autres soient nasillantes» (1972: 68). Ici, nous touchons au domaine de l'interprétation, riche en détails fascinants. Ainsi, par exemple, le même auteur donne une sorte de glossaire technique comprenant quelque soixantecinq termes touchant à la pratique musicale et constituant une base pour les critères d'appréciation (1972: 131-135). Avant de passer aux détails concernant l'interprétation, j'aimerais mentionner brièvement deux faits importants à propos des problèmes généraux du style.

12 Le premier a trait à une théorie d'Al-Fārābī sur les notes vides et les notes pleines, qui, de façon imagée, correspondrait à la dichotomie syllabique-mélismatique. La note vide est décrite comme un espace sonore limité par deux attaques successives et pouvant être meublé par des notes vocalisées. La note pleine est décrite comme un espace sonore rempli par les attaques répétées des lettres, un peu à la manière d'un ton récitatif. Il s'ensuit, sur le plan stylistique, deux espèces de chant: chant à notes vides et chant à notes pleines. Dans le premier, la mélodie en est plus belle, mais les paroles sont difficiles à comprendre; dans le second, les paroles sont aisément comprises, mais la mélodie en est moins belle et peu agréable. Al-Fārābī en conclut que le mieux serait un chant mixte, pour que l'on puisse trouver plaisir à sa mélodie et comprendre ses paroles (d'Erlanger 1935, vol. II: 66-68). 
13 Il semble, et ceci nous ramène au deuxième point, que cette approche se trouve à l'arrière-plan de la querelle entre Anciens et Modernes qui surgit au IX ${ }^{e}$ siècle et continue pendant l'âge d'or de la musique arabe. Les Anciens prêchaient la simplicité du chant et la mise en évidence des paroles; les Modernes tendaient vers l'exubérance et l'excès d'ornementation au risque d'entraîner un certain obscurcissement des paroles. Ainsi, alors que les Anciens aspiraient à établir un équilibre parfait entre texte et mélodie, les Modernes, en donnant plus de poids au composant mélodique aboutissaient, bon gré mal gré, à l'ébranlement de l'équilibre idéal.

\section{Critères d'excellence}

14 Nous arrivons maintenant au domaine le plus révélateur pour nous, qui est celui des jugements formulés à l'occasion de l'interprétation publique lors de compétitions et de débats organisés ou occasionnels. De façon schématique, nous pouvons résumer les éléments glanés dans différentes définitions qualifiant l'excellence du chanteur-musicien comme suit: le chanteur-musicien doit combiner une disposition naturelle pour la musique et un savoir bien poussé de sa théorie; avoir la faculté d'assimiler facilement ce qu'il entend et de le retenir dans sa mémoire, et d'être capable de transmettre la tradition avec précision dans un style personnel de préférence; avoir une belle voix, de l'expressivité, de la tendresse et la capacité de s'émouvoir et d'émouvoir.

15 Parmi les qualités spécifiques évoquées dans les textes, certaines ont trait à l'aspect physique, d'autre décrivent des capacités musicales. Ainsi par exemple, la beauté du visage, une taille bien formée, la parole douce et les belles manières sont considérées comme des facteurs susceptibles d'augmenter le plaisir musical. De même, il est recommandé que le chanteur ne fasse pas de grimaces, qu'il ne torde pas le cou, qu'il ne se penche pas, qu'il ne se balance pas, que les mouvements des yeux, des sourcils, de la main et de la tête soient discrets. Toutefois, les remarques les plus pertinentes soulignent la mise en évidence des capacités musicales distinguant le chanteur-musicien. Celles-ci comprennent un sentiment inné pour le rythme, l'usage correct des modes mélodiques et rythmiques, une bonne émission vocale, le maintien de la justesse des notes et le choix de la tessiture appropriée, une respiration bien dosée, la capacité de modifier un emprunt tout en évitant un plagiat évident, et le talent d'improviser. L'improvisation prévaut aussi bien au niveau d'une pièce individuelle que dans la conception et l'agencement d'un programme multi-sectionnel. Cette dernière caractéristique touche aussi aux rapports entre le musicien et son auditoire, qui est un sujet de prime importance. Il en est question déjà dans les premiers traités qui nous sont parvenus. C'est le cas par exemple d'Al-Kindi ( in Shiloah 1979: 254-255, 257) et des Frères sincères (in Shiloah 1978: 64-65), qui considèrent comme condition préalable exigée du musicien de connaitre le caractère de son auditeur et ses conditions psychologiques, ainsi que les circonstances régnantes, et de pouvoir y adapter son programme musical. Et pendant la séance, le musicien doit aussi sentir son auditeur et son humeur, et se conformer aux changements de ses états d'âme. Sur un plan plus sophistiqué, il est question dans ce genre de considérations des règles visant à éviter la monotonie et à recourir à des effets obéissant aux lois de contraste; c'est-à-dire faire suivre les moments de tension par des relâches; et, pour assurer le maintien de l'intérêt chez l'auditeur, de tenir compte des limitations imposées par la capacité de l'attention. Il est recommandé, par exemple, d'éviter ce qui est désagréable à l'ouverture d'une séance, et de la commencer avec les genres lourds des modes 
rythmiques et mélodiques, réservant les mélodies légères, rapides et stimulantes pour la conclusion de la séance. La transition d'un mode à l'autre, et d'un genre d'expression à un autre, doit se faire avec science et subtilité, et être bien préparée. Je pense que dans ces réflexions et recommandations se dessine, déjà au $\mathrm{X}^{\mathrm{e}}$ siècle, l'ébauche de la future forme composée qui sera réalisée sous les noms de nūba, fașil, maqām 'irāqī, waṣla, etc.

\section{Critères concernant l'auditeur}

Pour parvenir à la perfection, une séance musicale doit reposer sur l'interaction constante entre l'interprète et son public. L'auditeur avisé, assis face au musicien, doit posséder la faculté de saisir les qualités et les défauts de celui-ci, ainsi que de la musique qu'il interprète, et de prononcer son jugement adéquat sur le vif. Il existe à cet effet un ensemble de conventions déterminées par des interventions verbales que l'on énonce à l'audition d'un passage ou d'un détail frappant ou inopportun. Aux dires d'Al-Kātib, quand ce genre d'interventions est employé convenablement, elles seraient comparables "à l'éclat d'une perle »: «Cela revient à dire que si le chant comporte une perle, elle sera mise en évidence par le truchement des applaudissements, par la bienveillance que l'on montre en exigeant sa répétition et en faisant ressortir ses bonnes qualités. Si, par contre, le chant ne comporte pas de perle, il sera impossible d'y faire apparaître un quelconque complément ou d'y insuffler une certaine vie. » (1972: 181).

De ce bref tour d'horizon, qui n'a fait qu'effleurer un sujet qui mérite une étude approfondie, se dégage la conception de molécularité à laquelle nous avons fait allusion en la comparant avec celle qui prédomine dans la théorie littéraire. Toutefois, en dépit de l'attachement aux détails et de la fluidité des évaluations concernant la cohérence et l'unité de l'œuvre comme une entité strictement formalisée, on peut déceler un souci d'unité et de cohérence qui est sous-jacent. Nous l'avons remarqué par exemple à propos des séances musicales, où prédomine la recherche d'un enchaînement heureux d'éléments composant le tout, en tenant compte des lois de contraste et de variété en vue de conférer un sentiment de cohérence. Ces aspirations ont été à l'origine de la création des larges formes composées. A ce sujet, nous pouvons répéter, pour conclure, les propos de van Gelder: «Une séquence particulière d'associations peut paraître évidente et par conséquent cohérente à une personne mais pas à une autre, à une communauté littéraire mais pas à une autre ».

\section{BIBLIOGRAPHIE}

D’ERLANGER Rodolphe, 1935, La musique arabe. Tome II. Paris: Geuthner.

FARMER Henry George, 1957, "The music of Islam ", in: The New Oxford History of Music, vol. I: Ancient and Oriental Music. London: Oxford University Press, p. 421-477.

AL-KĀTIB Al-Ḥasan Ibn Aḥmad Ibn 'Alī, 1972, La perfection des connaissances musicales. Traduit et commenté par Amnon Shiloah. Paris: Geuthner. 
MERRIAM Alan P., 1964, The Anthropology of Music. Evanston (Ill.): Northwestern University Press.

SHILOAH Amnon, 1978, The Epistle on Music of the Ikhwān Al-Ṣafa. Tel-Aviv: Tel-Aviv University.

SHILOAH Amnon, 1979, The Theory of Music in Arabic Writings. Munich.

VAN GELDER Geert Jan H., 1982, Beyond the Line. Classical Arabic Literary Critics on the Coherence and Unity of the Poem. Leiden: E.J. Brill.

\section{RÉSUMÉS}

Dans son aperçu de «La musique de l'Islam (The New Oxford Hitory, vol. I, p. 433), Henry George Farmer dit: «La conception islamique ignorait l'esthétique en tant que science du beau. La raison principale était que l'Orient [....] avait une confiance inébranlable en le ta'thïr (influence) dans la musique, un dogme que les Grecs appelaient l'éthos». La doctrine de l'éthos exclue-t-elle réellement l'esthétique, la science du beau, ou plutôt le contredit-elle? En 1750, le Français Charles Fonton, en écrivant sur la musique turque, accusa ses contemporains de se laisser guider par l'amour-propre, par ce que nous appellerions aujourd'hui l'ethnocentrisme; il conclut: «La musique orientale possède sa beauté propre et ses propres critères pour l'évaluer ». Tout en essayant de tirer au clair l'approche de Farmer, on s'inspirera des écrits théoriques arabes afin d'évaluer l'approche de Fonton.

In his survey article "The Music of Islam» (The New Oxford History, vol. I, p. 433), H.G. Farmer states: "Aesthetics, the science of the beautiful, did not exist in Islamic conceptions. The chief reason for this was that the Orient [...] put great trust in the doctrine of the ta'thir (influence) in music, a dogma called by the Greeks the ethos». Does the doctrine of ethos really exclude aesthetics, the science of the beautiful, or is it in contradiction with it? In 1750, the Frenchman Charles Fonton, who wrote on Turkish music, accused his contemporaries of permitting themselves to be guided by self-love, which we would now call ethnocentrism, concluding: "Oriental music has its own beauty and its own criteria for judging that beauty ». While attempting to clarify Farmer's approach, evidence from the Arab theoretical writings will be provided to assess Fonton's approach.

\section{AUTEUR}

\section{AMNON SHILOAH}

Amnon Shiloah, né en 1928 et docteur de l'Université de Paris (Sorbonne), est actuellement professeur au Département de musicologie de l'Université hébraïque de Jérusalem. Auteur de nombreux articles, livres, monographies et disques, il a été professeur invité dans de nombreuses universités aux États-Unis et en Europe. Ses recherches portent sur divers aspects des musiques arabe et juive: histoire sociale et culturelle, édition scientifique, traduction commentée de traités musicaux, étude méthodologique et synthétique de traditions musicales vivantes. Ses principaux ouvrages sont: La perfection des connaissances musicales, The Musical Subjects in the Zohar, Jewish Iraqi Musical Tradition, Jewish Musical Traditions et The Theory of Music in Arab Writing. 